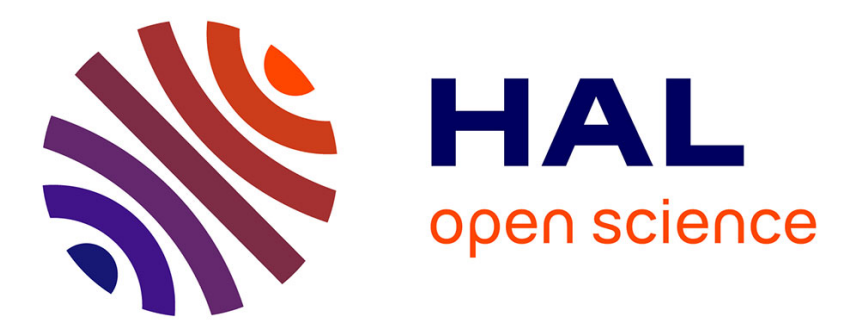

\title{
Leader-following consensus of second-order multi-agent systems with switching topology and partial aperiodic sampled data
}

Syed A Ajwad, Emmanuel Moulay, Michael Defoort, Tomas Menard, Patrick

Coirault

\section{To cite this version:}

Syed A Ajwad, Emmanuel Moulay, Michael Defoort, Tomas Menard, Patrick Coirault. Leaderfollowing consensus of second-order multi-agent systems with switching topology and partial aperiodic sampled data. IEEE Control Systems Letters, 2021, 5 (5), pp.1567-1572. 10.1109/LCSYS.2020.3041566 . hal-03086914

\section{HAL Id: hal-03086914 https://hal.science/hal-03086914}

Submitted on 23 Dec 2020

HAL is a multi-disciplinary open access archive for the deposit and dissemination of scientific research documents, whether they are published or not. The documents may come from teaching and research institutions in France or abroad, or from public or private research centers.
L'archive ouverte pluridisciplinaire $\mathbf{H A L}$, est destinée au dépôt et à la diffusion de documents scientifiques de niveau recherche, publiés ou non, émanant des établissements d'enseignement et de recherche français ou étrangers, des laboratoires publics ou privés. 


\title{
Leader-following consensus of second-order multi-agent systems with switching topology and partial aperiodic sampled data
}

\author{
Syed A. Ajwad, Emmanuel Moulay, Michael Defoort, Tomas Ménard and Patrick Coirault
}

\begin{abstract}
This article focuses on the problem of leaderfollowing consensus of second-order Multi-Agent Systems (MAS) with switching topology and partial aperiodic sampled data. MAS are subject to various constraints related to information exchange among the agents. It is considered that each agent in the network is able to measure its position only and cannot measure either its velocity or acceleration (input). Moreover, the position information is sent to the neighbors at aperiodic and asynchronous sampling rates. At last, a switching communication topology among the agents is considered. An observer-based control protocol is proposed to achieve leader-following consensus for MAS with above mentioned constraints. Using an Average Dwell Time (ADT) approach, sufficient conditions are derived through Lyapunov-based stability analysis to ensure the leader-following consensus. Numerical examples are also included to show the effectiveness of the proposed scheme.
\end{abstract}

Index Terms-Average dwell time, Continuous-discrete time observer, Leader-follower consensus, Nonuniform and asynchronous sampling, Switching graphs

\section{INTRODUCTION}

$\mathbf{T}$ HE consensus problem in Multi-Agent Systems (MAS) has attracted enormous attention of research communities from various fields like biology, physics, computing, control engineering and robotics. Broadly speaking, consensus means that the agents reach an agreement on some common value of state called the consensus state. In leader-following consensus, the agents are not only required to reach a common state but also to track a desired trajectory generated by a leader whose dynamics is independent of the followers.

Information exchange among the agents is crucial in cooperative control. Advancements in computing and communication technologies have enabled agents to exchange information directly with each other without any central system. This allows researchers to design distributed cooperative controllers

S. A. Ajwad and P. Coirault are with the Université de Poitiers, LIAS (EA 6315), 2 rue Pierre Brousse, 86073 Poitiers, France (e-mail: syed.ali.ajwad@univ-poitiers.fr, patrick.coirault@univ-poitiers.fr).

E. Moulay is with the Université de Poitiers, XLIM (UMR CNRS 7252), 11 bd Marie et Pierre Curie, 86073 Poitiers, France (e-mail: emmanuel.moulay@univ-poitiers.fr).

M. Defoort is with the Universite Polytechnique Hauts-de-France, INSA, LAMIH (UMR CNRS 8201), Le Mont Houy, 59313 Valenciennes, France (e-mail: michael.defoort@uphf.fr).

T. Ménard is with the Université de Caen, LAC (EA 7478), 6 bd du Maréchal Juin, 14032 Caen, France (e-mail: tomas.menard@unicaen.fr). which provide better efficiency, reliability and scalability as compared to centralized controllers [1].

The distributed consensus problem for first-order MAS has been extensively studied, for instance in [2], [3]. However, the results for first-order MAS cannot be directly applied for second-order MAS where agents' dynamics are governed by position and velocity. Since many physical systems can be characterized by second-order dynamics, various distributed control algorithms for second-order MAS have been proposed in literature to deal with both leaderless and leader-following consensus problems [4], [5], [6], [7].

It should be noted that most of these results consider that the communication among the agents is continuous and fixed. Moreover, it is often assumed that the agents can measure and transmit both their position and velocity states. However, these considerations are not valid for real engineering MAS applications. It is sometimes difficult to measure all states which are not even desirable due to related cost and compact sizes of the agents. Moreover, since the communication and computing equipment are digital, the information exchange between the agents cannot be continuous. Several control techniques have been proposed by the research community to deal with the consensus problem of MAS with discrete data [8], [9]. Consensus problem with partial available data is also discussed [10], [11], [12]. In [10], Xu et al. proposed a discontinuous observer-based leader-following protocol. Yu et al. introduced a consensus protocol by using current and previous samples of position data [11]. The authors of [12] gave necessary and sufficient conditions to achieve consensus in MAS with sampled position information. However, in these articles, the sampling period is considered constant. In [13], [14], consensus algorithms have been investigated for MAS with stochastic sampling periods. However, in practical applications, the sampling could be arbitrary non-uniform as well as asynchronous where each agent has independent sampling time from the other agents in the network. Moreover, in MAS with discrete data transmission, the input is mostly kept constant. However, one can achieve continuous control input by reconstructing the state in continuous time from the discrete information. This can be obtained by using continuous-discrete time observer [15]. In [16] and [17] such observer has been used to design leaderless and leader-following control protocols respectively. However, in these articles, it is considered that the communication topology among the agents remains constant. On the other hand, it is sometimes not feasible for 
the agents to maintain a fixed communication topology due to various reasons like collision avoidance, communication link failure or communication range limitations etc. Therefore, in these scenarios, it is mandatory to consider switching topology. Consensus of MAS with switching topology has been discussed widely in literature e.g. [18], [19]. However, these articles do not consider the above mentioned communication constraints of irregular and asynchronous sampling and unavailability of velocity state.

Motivated by the above discussion, in this paper, we propose a distributed leader-following consensus algorithm for second-order MAS with switching interaction topology and communication constraints. It is considered that each agent only transmits its position state to its neighbors with arbitrary non-uniform and asynchronous sampling periods. The velocity and acceleration are unavailable. Furthermore, the leader sends its position information to only a small group of followers in the network. The communication among the agents is directed. It must be noted that as compared to [17], where only fixed communication topology is considered, we assume that the interaction topology among the agents does not remain constant and changes with time. Due to the switching topology, the stability analysis becomes more complex. It should be noted that the results of [17] cannot be directly applied for the case of switching topology since switching topology can make the overall system unstable. In this paper, using an Average Dwell Time (ADT) approach, sufficient conditions are derived through Lyapunov-based stability analysis to ensure the leaderfollowing consensus.

The remaining paper is organized as follows. Preliminaries on graph theory are provided in Section II followed by the problem statement in Section III. Main results are presented in Section IV, Section V contains numerical examples and the article is concluded in Section VI.

\section{RECAlLs ON GRAPH THEORY}

A directed graph $\mathcal{G}$ is a pair $(\mathcal{V}, \mathcal{E})$. The set of agents $\mathcal{V}$ is nonempty and finite. The set of edges $\mathcal{E} \subseteq \mathcal{V} \times \mathcal{V}$ denotes ordered pairs of distinct agents. $(i, j) \in \mathcal{E}$ if agent $j$ can receive information from agent $i$. A graph has a directed spanning tree if there exists a directed path from the root to all other agents. For a graph $\mathcal{G}$ with $N$ agents, the adjacency matrix $\mathcal{A}=\left(a_{i j}\right) \in \mathbb{R}^{N \times N}$ with $a_{i j}=1$ if agent $i$ can receive information from agent $j$ and $a_{i j}=0$ otherwise; and the Laplacian matrix $\mathcal{L}=\left(l_{i j}\right) \in \mathbb{R}^{N \times N}$ as $l_{i i}=$ $\sum_{j \neq i} a_{i j}, l_{i j}=-a_{i j}$ for $i \neq j$. Considering the directed graph combining both the leader and the followers, denoted by $\overline{\mathcal{G}}$, one can define the pinning matrix as the diagonal matrix $\mathcal{B}=\operatorname{diag}\left(b_{1}, b_{2}, \ldots, b_{N}\right)$ with $b_{j}=1$ if follower $j$ can receive information from the leader and $b_{j}=0$ otherwise.

\section{Problem statement}

Let us consider a MAS which consists of $N$ followers with the following dynamics

$$
\dot{x}_{i}(t)=A x_{i}(t)+B u_{i}(t), \quad y_{i}(t)=C x_{i}(t)
$$

for $i=1, \ldots, N$, the state is $x_{i}(t)=\left[r_{i}(t)^{T}, v_{i}(t)^{T}\right]^{T}$ where $r_{i}(t) \in \mathbb{R}^{m}$ is the position while $v_{i}(t) \in \mathbb{R}^{m}$ is the velocity of agent $i$. The control input of agent $i$ is $u_{i} \in \mathbb{R}^{m} \cdot y_{i} \in$ $\mathbb{R}^{m}$ denotes the measured position for agent $i$. Note that the position data are transmitted between agents according to the communication topology in a discrete aperiodic way. $A=$ $\left(\begin{array}{cc}0_{m} & I_{m} \\ 0_{m} & 0_{m}\end{array}\right), B=\left(\begin{array}{l}0_{m} \\ I_{m}\end{array}\right)$ and $C=\left(\begin{array}{ll}I_{m} & 0_{m}\end{array}\right)$ are the system input and output matrices, respectively. The leader agent has the following dynamics

$$
\dot{x}_{0}(t)=A x_{0}(t), \quad y_{0}(t)=C x_{0}(t)
$$

where $x_{0}$ is the leader state and $y_{0}$ is the measured position for the leader.

Definition 1: The leader-following consensus of MAS (1)(2) is achieved if $\lim _{t \rightarrow \infty}\left\|x_{i}(t)-x_{0}(t)\right\|=0, i=1 \ldots N$.

It is considered that each agent in the network only transmits its position $r_{i}$ to its neighbours at aperiodic and asynchronous time instants. The velocity $v_{i}$ and the input/acceleration $u_{i}$ are completely unavailable. Let $t_{k}^{i, j}$ be the time instant at which agent $j$ sends its position data to agent $i$ with $i=1, \ldots, N$, $j=0, \ldots, N(j \neq i)$ and $k \in \mathbb{N}$. Moreover, there exist two constants $\tau_{m} \geq 0, \tau_{M}>0$ called minimum and maximum sampling time respectively such that $\tau_{m}<t_{k+1}^{i, j}-t_{k}^{i, j}<\tau_{M}$.

Denote $\underline{\mathcal{G}}=\left\{\overline{\mathcal{G}}^{1}, \overline{\mathcal{G}}^{2}, \ldots, \overline{\mathcal{G}}^{M}\right\}$ as a finite set of possible topology graphs and $\mathcal{M}=\{1,2, \ldots, M\}$ represents the set of indices. Each graph in $\underline{\mathcal{G}}$ has the same nodes (agents) but can have different edges. The switching between the graphs is time dependant and is modelled by a switching function $\sigma(t):[0, \infty) \rightarrow \mathcal{M}$ which is a piece-wise constant function, determining the topology of the dynamic network at each time instant. In this paper, it is assumed that $\sigma(t)$ is generated exogenously and satisfies the minimum dwell time condition to avoid chattering and Zeno behavior in the network dynamics. Let $0=t_{0}<t_{1}<t_{2} \ldots$ be the switching instants of $\sigma(t)$. Furthermore, the intervals $\left(t_{l}, t_{l+1}\right], l=0,1, \ldots$ are bounded and contiguous. Denote the directed switching graph as $\mathcal{G}^{\sigma(t)} \in \underline{\mathcal{G}}$ with $A^{\sigma(t)}$ and $L^{\sigma(t)}$ the corresponding adjacency and Laplacian matrices respectively. Let us denote the diagonal matrix $\mathcal{B}^{\sigma(t)}=\operatorname{diag}\left(b_{1}^{\sigma}(t), \ldots, b_{N}^{\sigma}(t)\right)$ which represents the switching interconnection between the leader and the followers. $b_{i}^{\sigma}(t)$, for $i=1, \ldots, N$ is equal to 1 if agent $i$ can receive information from the leader and zero otherwise. The switching communication graph including the followers and the leader is denoted $\overline{\mathcal{G}}^{\sigma(t)}$.

Assumption 1: Each switching graph $\overline{\mathcal{G}}^{\sigma(t)}$ has a directed spanning tree with the leader as a root.

Let us define matrix

$$
\mathcal{H}^{\sigma(t)}=\mathcal{L}^{\sigma(t)}+\mathcal{B}^{\sigma(t)}
$$

If graph $\overline{\mathcal{G}}^{\sigma(t)}$ has a directed spanning tree, then $\mathcal{H}^{\sigma(t)}$ is a nonsingular M-matrix [20] and there exists a diagonal matrix $\Omega^{\sigma}=\operatorname{diag}\left(\omega_{1}^{\sigma}, \ldots, \omega_{N}^{\sigma}\right)$ such that [21]

$$
\mathcal{H}^{\sigma T} \Omega^{\sigma}+\Omega^{\sigma} \mathcal{H}^{\sigma}>0 .
$$

Define the following notations

$$
\begin{aligned}
\omega_{\max }^{\sigma} & =\max \left\{\omega_{1}^{\sigma}, \ldots, \omega_{N}^{\sigma}\right\}, \\
\rho^{\sigma} & =\lambda_{\min }\left(\mathcal{H}^{\sigma T} \Omega^{\sigma}+\Omega^{\sigma} \mathcal{H}^{\sigma}\right) .
\end{aligned}
$$


where $\lambda_{\min }($.$) denotes the smallest eigenvalue. The control$ objective is to design distributed consensus protocols $u_{i}(i=$ $1, \ldots, N)$, based on available aperiodic and asynchronous sampled position data such that leader-following consensus on switched dynamic network $\overline{\mathcal{G}}^{\sigma(t)}$ ( $\sigma$ satisfies the minimum dwell time condition) is achieved according to Definition 1 . To solve this problem, let us recall the following useful definition.

Definition 2 ([22]): For any switching signal $\sigma(t)$ and $t_{2}>$ $t_{1} \geq t_{0}$, let $N_{\sigma\left(t_{2}, t_{1}\right)}$ describes the number of switching of $\sigma(t)$ over the time interval $\left[t_{1}, t_{2}\right)$. For any $\tau_{a}>0$ and an integer $N_{0} \geq 0$, if

$$
N_{\sigma\left(t_{2}, t_{1}\right)}<N_{0}+\frac{t_{2}-t_{1}}{\tau_{a}}
$$

holds, then $\tau_{a}$ is called the Average Dwell Time (ADT).

Lemma 1: [17] If $v_{1}(t)$ and $v_{2}(t)$ are real valued functions verifying $\frac{d}{d t}\left(v_{1}^{2}(t)+v_{2}^{2}(t)\right) \leq-a v_{1}^{2}(t)-b v_{2}^{2}(t)+$ $c \int_{t-\delta}^{t} v_{2}^{2}(s) d s+k$ for $t \geq 0$, where $a, b, \delta>0$ and $c, k \geq 0$. There exist $\varrho>0$ and $\bar{\alpha} \geq 0$ such that if $\delta<\varrho$, then $v_{1}^{2}(t)+v_{2}^{2}(t) \leq \bar{\alpha} e^{-\sigma t}+\frac{k}{\sigma}, \forall t \geq 0$ where $\sigma$ is given by $\sigma=\frac{1}{2} \min (a, b)$.

\section{MAIN RESULTS}

The proposed distributed control law is given as follows

$$
\begin{aligned}
u_{i}(t)= & -\bar{c} K_{c} \Gamma_{\lambda} \sum_{j=1}^{N} a_{i j}^{\sigma(t)}\left[\hat{x}_{i, i}(t)-\hat{x}_{i, j}(t)\right] \\
& -b_{i}^{\sigma(t)} \bar{c} K_{c} \Gamma_{\lambda}\left[\hat{x}_{i, i}(t)-\hat{x}_{i, 0}(t)\right]
\end{aligned}
$$

for $i=1, \ldots, N$ where $a_{i j}^{\sigma(t)}$ is the $i j^{\text {th }}$ entry of adjacency matrix $A^{\sigma(t)}, \bar{c}$ is the coupling strength, $K_{c}=B^{T} Q=$ $\left(\begin{array}{ll}I_{m} & 2 I_{m}\end{array}\right)$ with $Q$ the symmetric positive definite matrix solution of algebraic Lyapunov equation [23]

$$
Q+Q A+A^{T} Q=Q B B^{T} Q
$$

and $\Gamma_{\lambda}=\left(\begin{array}{cc}\lambda^{2} I_{m} & 0_{m} \\ 0_{m} & \lambda I_{m}\end{array}\right)$ where $\lambda$ is the controller tuning parameter. One should note that as compared to the control input proposed in [17], $a_{i j}^{\sigma(t)}$ in (8) is not constant but changes with the communication graph $\overline{\mathcal{G}}^{\sigma(t)} \cdot \hat{x}_{i, j}(t)=\left[\hat{r}_{i, j}^{T}(t), \hat{v}_{i, j}^{T}(t)\right]^{T}$, $i=1, \ldots, N, j=0, \ldots, N$ where $\hat{r}_{i, j}(t)$ and $\hat{v}_{i, j}(t)$ are the estimation of position and velocity respectively of agent $j$ estimated by agent $i$ from the available aperiodic and asynchronous sampled position data. They are computed as follows:

$$
\begin{aligned}
\dot{\hat{x}}_{i, j}(t)= & A \hat{x}_{i, j}(t)-\theta \Delta_{\theta}^{-1} K_{o} e^{-2 \theta\left(t-\kappa_{i, j}(t)\right)}\left[\hat{r}_{i, j}\left(\kappa_{i, j}(t)\right)\right. \\
& \left.-r_{j}\left(\kappa_{i, j}(t)\right)\right]
\end{aligned}
$$

where $\theta$ represents the observer tuning parameter while $\Delta_{\theta}=$ $\left(\begin{array}{cc}I_{m} & 0_{m} \\ 0_{m} & \frac{1}{\theta} I_{m}\end{array}\right)$ and $K_{o}=P^{-1} C^{T}=\left[\begin{array}{ll}2 I_{m} & I_{m}\end{array}\right]^{T}$, with $P$ the symmetric positive definite matrix solution of the algebraic Lyapunov equation [24]

$$
P+A^{T} P+P A=C^{T} C
$$

$\kappa_{i, j}(t)=\max \left\{t_{k}^{i, j} \mid t_{k}^{i, j} \leq t, k \in \mathbb{N}\right\}$ is the last instant when agent $i$ receives the position data of agent $j$. One can note that observer (10) represents a high-gain continuous-discrete time observer which estimates the state of an agent and its neighbors in continuous time from sampled aperiodic and asynchronous position information. Furthermore, since a timevarying exponential gain is used and the correcting term in the observer consists of both continuous and discrete parts, the observer dynamics (10) is hybrid and nonlinear.

Assumption 2: At each switching instant $t_{l}, l=0,1, \ldots$, every agent of the MAS sends its own estimated states, $\hat{r}_{i, i}\left(t_{l}\right), \hat{v}_{i, i}\left(t_{l}\right)$, with $i=0, \ldots, N$, to its new neighbors. The observer updates its value at time $t=t_{l}$ based on the estimations it receives from the neighbors, i.e. $\hat{r}_{i, j}\left(t_{l}\right)=$ $\hat{r}_{j, j}\left(t_{l}\right)$ and $\hat{v}_{i, j}\left(t_{l}\right)=\hat{v}_{j, j}\left(t_{l}\right)$.

Remark 1: Assumption 2 is important for the convergence of the observer in the case of switching graphs. It ensures that once the observer error reaches zero, it will not diverge due to switching between graphs. Also, other than switching instants, i.e. when $t \neq t_{l}$, the observer dynamics are governed by (10). At last, the same observer can be used by a real leader to estimate its own states which could be transmitted to its neighbors at the switching instant.

Theorem 1: Consider the MAS (1)-(2) with control input (8) and let Assumptions 1 and 2 hold. If the control parameters $\theta, \lambda, \bar{c}>0$ satisfy the following

$$
\begin{aligned}
\theta & <\frac{\bar{\varrho}}{\tau_{M}} \\
\lambda & <\epsilon^{*} \theta \\
\bar{c} & \geq \frac{\max _{p \in \mathcal{M}}\left\{\omega_{\max }^{p}\right\}}{\min _{p \in \mathcal{M}}\left(\rho^{p}\right)}
\end{aligned}
$$

where $\bar{\varrho}$ is a positive constant, $\epsilon^{*} \in(0,1), \omega_{\max }^{p}$ and $\rho^{p}$ are given by (5) and (6) respectively, and if the ADT satisfies the following inequality

$$
\tau_{a}>\frac{8 \ln (\beta K)-1}{\lambda}
$$

with $K, \beta \geq 1$ are constants, then the leader-following consensus is achieved under switching dynamic network.

Remark 2: The proposed leader-following algorithm is distributed since it only requires position information of the neighbors i.e only local information. Moreover, there is no centralized unit to calculate the the input of the agents. Instead each agent is computing its own input based on the discrete information it receives from the neighbors. One may remark that algorithm requires the position information in the global frame or the tuning parameters require information of communication topology. However, these are very common assumptions in designing of distributed algorithms for MAS, see for example [25], [26]. Nevertheless, the tuning gains are tuned beforehand and remain constant for all $t \geq 0$ and then only local information is used to compute the control input.

Proof: The proof of Theorem 1 is divided into three steps. In the first step, we consider the case of fixed topology and obtain some useful results. Then in the second step, some important results are derived for switching topology. Finally in step 3, all the flows and jumps related to switching topology are combined using previously obtained results and a condition of ADT is achieved to ensure the stability of the system. 
Step 1: Consider a fixed communication graph $\mathcal{G}^{p}(p \in \mathcal{M})$. Let us define the estimation error as

$$
\tilde{x}_{i, j}(t)=\hat{x}_{i, j}(t)-x_{j}(t), \quad j=0, \ldots, N
$$

and the tracking error as

$$
e_{i}(t)=x_{i}(t)-x_{0}(t), \quad i=1, \ldots N .
$$

Consider the new coordinates for classical high-gain design $\bar{e}_{i}=\Gamma_{\lambda} e_{i}$ and $\bar{x}_{i, j}=\Delta_{\theta} \tilde{x}_{i, j}$. Denoting $\eta^{c}=\left[\bar{e}_{1}^{T} \ldots \bar{e}_{N}^{T}\right]^{T}$, $\eta_{i}^{o}=\left[\left(\bar{x}_{i, 1}\right)^{T} \ldots\left(\bar{x}_{i, N}\right)^{T}\right]^{T}$ for $i=1 \ldots N$ and $\eta_{0}^{o}=$ $\left[\left(\bar{x}_{1,0}\right)^{T} \ldots\left(\bar{x}_{N, 0}\right)^{T}\right]$. Consider the following candidate Lyapunov functions

$$
\begin{aligned}
\bar{V}_{c}^{p}\left(\eta^{c}(t)\right) & =\left(\eta^{c}(t)\right)^{T}\left[\Omega^{p} \otimes Q\right] \eta^{c}(t) \\
V_{o}\left(\bar{x}_{i, j}(t)\right) & =\left(\bar{x}_{i, j}(t)\right)^{T} P \bar{x}_{i, j}(t) \\
\bar{V}_{o}\left(\eta^{o}(t)\right) & =\sum_{i=1}^{N} \sum_{j=0}^{N} V_{o}\left(\bar{x}_{i, j}(t)\right)
\end{aligned}
$$

Taking the derivative of the above Lyapunov functions and applying Lemma 1, the following inequality is achieved if conditions (12)-(14) and Assumption 1 are satisfied (please see [17] for more details)

$$
\sqrt{\bar{V}_{c}^{p}\left(\eta^{c}(t)\right)}+\epsilon^{\frac{3}{2}} \theta^{2} \sqrt{\bar{V}_{o}\left(\eta^{o}(t)\right)} \leq \bar{\alpha}\left(t_{0}\right) e^{-\frac{\lambda}{8}\left(t-t_{0}\right)}
$$

where $\bar{\alpha}\left(t_{0}\right)$ is given as

$$
\begin{aligned}
\bar{\alpha}\left(t_{0}\right)= & \sqrt{\bar{V}_{c}^{p}\left(\eta^{c}\left(t_{0}\right)\right)}+\epsilon^{\frac{3}{2}} \theta^{2} \sqrt{\bar{V}_{0}\left(\eta^{o}\left(t_{0}\right)\right)}+c \epsilon^{\frac{3}{2}} \theta^{2} \\
& \times \int_{0}^{\tau_{M}} \int_{t_{0}-s}^{t_{0}} e^{v \kappa\left(\mu-t_{0}+s\right)} \sqrt{\bar{V}_{0}(\mu)} d \mu d s
\end{aligned}
$$

where $c>0, v \geq 0$ and $\kappa \geq 0$ (see Lemma 3 of [17] for details).

It is clear from (21) that the system achieves stability for a fixed communication topology. However, this does not imply that the closed-loop system will remain stable in the case of switching communication topology since switching may lead to an overall unstable system. Therefore, we need to find out the stability conditions considering switching topology. We first need the following results. Considering time interval $t \in$ $\left[t_{0}, t_{1}\right)$ and using (22), (21) can be written as:

$$
\begin{aligned}
& \sqrt{\bar{V}_{c}^{p}\left(\eta^{c}(t)\right)}+\epsilon^{\frac{3}{2}} \theta^{2} \sqrt{\bar{V}_{0}(t)} \\
& \leq\left(\sqrt{\bar{V}_{c}^{p}\left(\eta^{c}\left(t_{0}\right)\right)}+\epsilon^{\frac{3}{2}} \theta^{2} K \max _{s \in\left[t_{0}-\tau_{M}, t_{0}\right]} \sqrt{\bar{V}_{o}(s)}\right) e^{-\frac{\lambda}{8}\left(t-t_{0}\right)}
\end{aligned}
$$

so one can obtain the following

$$
\begin{aligned}
& \sqrt{\bar{V}_{c}^{p}\left(\eta^{c}\left(t_{1}\right)\right)}+\epsilon^{\frac{3}{2}} \theta^{2} \max _{s \in\left[t_{1}-\tau_{M}, t_{1}\right)} \sqrt{\bar{V}_{0}(s)} \\
\leq & \left(\sqrt{\bar{V}_{c}^{p}\left(\eta^{c}\left(t_{0}\right)\right)}+\epsilon^{\frac{3}{2}} \theta^{2} K \max _{s \in\left[t_{0}-\tau_{M}, t_{0}\right]} \sqrt{\bar{V}_{o}(s)}\right) \\
& \times\left(e^{\frac{\lambda}{8} \tau_{M}}\right) e^{-\frac{\lambda}{8}\left(t_{1}-t_{0}\right)}
\end{aligned}
$$

with $K=\max \left\{1, c \tau_{M}^{2} e^{v \kappa \tau_{M}}\right\} . \bar{V}_{c}^{p}$ satisfies the following properties [27], [28]:

- Since $\omega^{i} \otimes Q$ is always symmetric positive definite for any $i \in \mathcal{M}$, there exists $\bar{\beta} \geq 1$ such that

$$
\bar{V}_{c}^{p} \leq \bar{\beta} \bar{V}_{c}^{q}, \quad \forall p, q \in \mathcal{M}
$$

so

$$
\sqrt{\bar{V}_{c}^{p}} \leq \beta \sqrt{\bar{V}_{c}^{q}}, \quad \forall p, q \in \mathcal{M}
$$

where $\beta=\sqrt{\bar{\beta}}$;

- Let $\alpha_{1}=\min _{p \in \mathcal{M}}\left(\lambda_{\min }\left(\Omega^{p} \otimes Q\right)\right)$ and $\alpha_{2}=$ $\max _{p \in \mathcal{M}}\left(\lambda_{\max }\left(\Omega^{p} \otimes Q\right)\right)$, then

$$
\alpha_{1}\left\|\eta^{c}\right\|^{2} \leq \bar{V}_{c}^{p} \leq \alpha_{2}\left\|\eta^{c}\right\|^{2} .
$$

Step 2: Let us now define a piecewise Lyapunov function for the considered switching communication topology

$$
\bar{V}_{c}^{\sigma(t)}\left(\eta^{c}(t)\right)=\left(\eta^{c}(t)\right)^{T}\left[\Omega^{\sigma(t)} \otimes Q\right] \eta^{c}(t) .
$$

Then, from (26), for any switching instant $t_{l}, l=1,2, \ldots$, one can get

$$
\sqrt{\bar{V}_{c}^{\sigma\left(t_{l}\right)}\left(\eta^{c}\left(t_{l}\right)\right)} \leq \beta \sqrt{\bar{V}_{c}^{\sigma\left(t_{l}^{-}\right)}\left(\eta^{c}\left(t_{l}^{-}\right)\right)} .
$$

Furthermore, if Assumption 2 is satisfied, one has

$$
\bar{V}_{0}\left(\eta^{o}\left(t_{l}\right)\right)=\bar{V}_{0}\left(\eta^{o}\left(t_{l}^{-}\right)\right) .
$$

Hence, one can obtain

$$
\begin{aligned}
& \left(\sqrt{\bar{V}_{c}^{\sigma\left(t_{l}\right)}\left(\eta^{c}\right)}+\epsilon^{\frac{3}{2}} \theta^{2} \max _{s \in\left[t_{l}, t_{l}-\tau_{M}\right)} \sqrt{\bar{V}_{o}(s)}\right) \\
\leq & \beta\left(\sqrt{\bar{V}_{c}^{\sigma\left(t_{l}^{-}\right)}\left(\eta^{c}\right)}+\epsilon^{\frac{3}{2}} \theta^{2} \max _{s \in\left[t_{l}^{-}, t_{l}^{-}-\tau_{M}\right)} \sqrt{\bar{V}_{o}(s)}\right)
\end{aligned}
$$

Step 3: For $t \in\left[t_{k}, t_{k+1}\right)$, from (23) and (31), one has

$$
\begin{aligned}
& \sqrt{\bar{V}_{c}^{\sigma(t)}\left(\eta^{c}\right)}+\epsilon^{\frac{3}{2}} \theta^{2} \max _{s \in\left[t-\tau_{M}, t\right)} \sqrt{\bar{V}_{0}(s)} \\
\leq & e^{\frac{\lambda}{8} \tau_{M}}\left(\sqrt{\bar{V}_{c}^{\sigma\left(t_{k}\right)}\left(\eta^{c}\right)}\right. \\
& \left.+\epsilon^{\frac{3}{2}} \theta^{2} K \max _{s \in\left[t_{k}-\tau_{M}, t_{k}\right]} \sqrt{\bar{V}_{o}(s)}\right) e^{-\frac{\lambda}{8}\left(t-t_{k}\right)} \\
\leq & \beta K e^{\frac{\lambda}{8} \tau_{M}}\left(\sqrt{\bar{V}_{c}^{\sigma\left(t_{k}^{-}\right)}\left(\eta^{c}\right)}\right. \\
& \left.+\epsilon^{\frac{3}{2}} \theta^{2} \max _{s \in\left[t_{k}^{-}-\tau_{M}, t_{k}^{-}\right]} \sqrt{\bar{V}_{o}(s)}\right) e^{-\frac{\lambda}{8}\left(t-t_{k}\right)} \\
\leq & \beta K^{2} e^{2 \frac{\lambda}{8} \tau_{M}}\left(\sqrt{\bar{V}_{c}^{\sigma\left(t_{0}\right)}\left(\eta^{c}\left(t_{k-1}\right)\right)}\right. \\
& \left.+\epsilon^{\frac{3}{2}} \theta^{2} \max _{s \in\left[t_{k-1}-\tau_{M}, t_{k-1}\right]} \sqrt{\bar{V}_{o}(s)}\right) e^{-\frac{\lambda}{8}\left(t-t_{k-1}\right)} \\
\leq & \beta^{N_{\sigma}}\left(K e^{\frac{\lambda}{8} \tau_{M}}\right)^{N_{\sigma}+1}\left(\sqrt{\bar{V}_{c}^{\sigma\left(t_{0}\right)}\left(\eta^{c}\right)}\right. \\
& \left.+\epsilon^{\frac{3}{2}} \theta^{2} \max _{s \in\left[t_{0}-\tau_{M}, t_{0}\right]} \sqrt{\bar{V}_{o}(s)}\right) e^{-\frac{\lambda}{8}\left(t-t_{0}\right)} \\
\leq & \beta^{N_{0}}\left(K e^{\frac{\lambda}{8} \tau_{M}}\right)^{N_{0}+1}(\beta K)^{\frac{t-t_{0}}{\tau_{a}}} e^{\frac{t-t_{0}}{\tau_{a}}}\left(\sqrt{\bar{V}_{c}^{\sigma\left(t_{0}\right)}\left(\eta^{c}\right)}\right. \\
& \left.+\epsilon^{\frac{3}{2}} \theta^{2} \max _{s \in\left[t_{0}-\tau_{M}, t_{0}\right]} \sqrt{\bar{V}_{o}(s)}\right) e^{-\frac{\lambda}{8}\left(t-t_{0}\right)}
\end{aligned}
$$


Now, using Definition 2, one has

$$
\begin{aligned}
& \sqrt{\bar{V}_{c}^{\sigma(t)}\left(\eta^{c}\right)}+\epsilon^{\frac{3}{2}} \theta^{2} \max _{s \in\left[t-\tau_{M}, t\right)} \sqrt{\bar{V}_{0}(s)} \\
\leq & \beta^{N_{0}}\left(K e^{\frac{\lambda}{8} \tau_{M}}\right)^{N_{0}+1}\left(\sqrt{\bar{V}_{c}^{\sigma\left(t_{0}\right)}\left(\eta^{c}\right)}\right. \\
& \left.+\epsilon^{\frac{3}{2}} \theta^{2} \max _{s \in\left[t_{0}-\tau_{M}, t_{0}\right]} \sqrt{\bar{V}_{o}(s)}\right) e^{-\left(\frac{\lambda}{8}-\frac{\ln (\beta K)-1}{\tau_{a}}\right)\left(t-t_{0}\right)}
\end{aligned}
$$

Hence, if the ADT satisfies condition (15), system (1)-(2) achieves leader-following consensus under switching dynamic network. From the original coordinates of tracking error, we can achieve the following inequality:

$$
\sum_{i=1}^{N}\left\|e_{i}\right\| \leq l_{1} \sqrt{\bar{V}_{c}^{\sigma(t)}\left(\eta^{c}\right)}
$$

with $l_{1}=\frac{1}{\lambda} \frac{\sqrt{N}}{\sqrt{\lambda_{\min }(Q)} \sqrt{\omega_{\min }^{\sigma(t)}}}$. From the over-valuation of (34) and using (35), we achieve

$$
\sum_{i=1}^{N}\left\|e_{i}\right\| \leq l_{1} \bar{\gamma} e^{-\left(\frac{\lambda}{8}-\frac{\ln (\beta K)-1}{\tau_{a}}\right)\left(t-t_{0}\right)}
$$

where $\bar{\gamma}=\beta^{N_{0}}\left(K e^{\frac{\lambda}{8} \tau_{M}}\right)^{N_{0}+1}\left(\sqrt{\bar{V}_{c}^{\sigma\left(t_{0}\right)}\left(\eta^{c}\right)}+\epsilon^{\frac{3}{2}} \theta^{2}\right.$

$\left.\max _{s \in\left[t_{0}-\tau_{M}, t_{0}\right]} \sqrt{\bar{V}_{o}(s)}\right)$. It is clear from (36) that if ADT satisfies condition (15), the tracking error decays exponentially.

\section{SiMULATIONS}

We consider a MAS with 4 followers labelled from 1 to 4 and a leader labelled 0 for simulation purposes. Three possible communication topologies are shown in Figure 1. The topologies are switching according to the switching signal
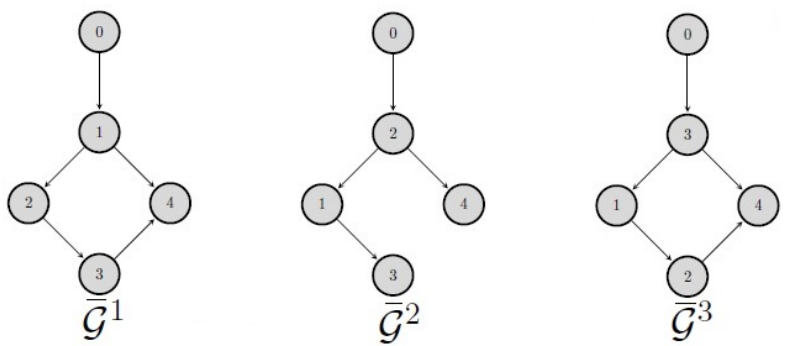

Fig. 1: Communication graphs

shown in Figure 2. The minimum dwell time between two consecutive switchings is chosen to be equal to $1 \mathrm{~s}$. Position

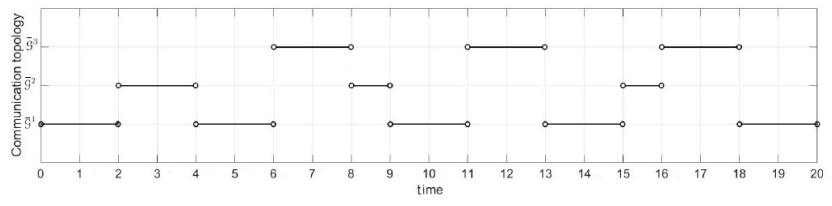

Fig. 2: Switching signal

data is transmitted between neighboring agents in an aperiodic and asynchronous discrete way. The associated minimum sampling time $\tau_{m}$ is $0.01 \mathrm{~s}$ while the maximum sampling time $\tau_{M}$ is $0.15 \mathrm{~s}$. The initial conditions of the agents are selected randomly. The control gains are tuned and set as $\bar{c}=1.2$, $\lambda=0.8$ and $\theta=12$.

In the first case, the leader is kept stationary. Figure 3-(a) shows the position consensus while the velocity consensus is shown in Figure 3-(b). The corresponding errors are depicted

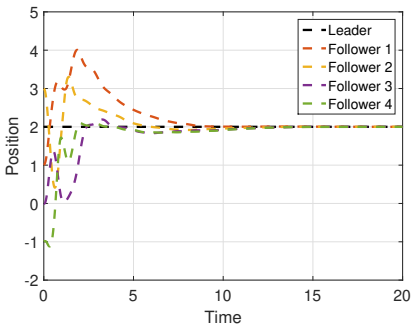

(a)

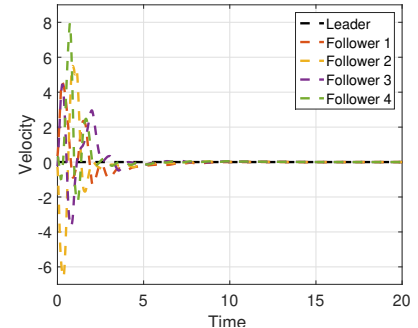

(b)
Fig. 3: Leader following consensus with a stationary leader (a) position consensus - (b) velocity consensus

in Figure 4. In the second simulation, the leader moves with
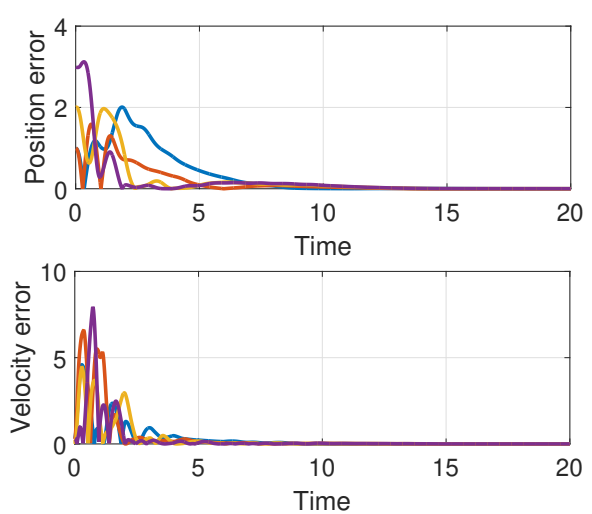

Fig. 4: Tracking errors for consensus with a stationary leader

a velocity of $1.5 \mathrm{~m} . \mathrm{s}^{-1}$. Figure 5 shows the results for both position and velocity consensus while the tracking errors are depicted in Figure 6. An example of sampling instants

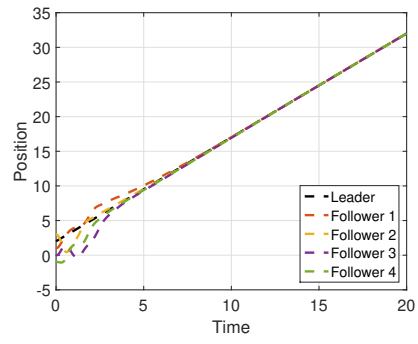

(a)

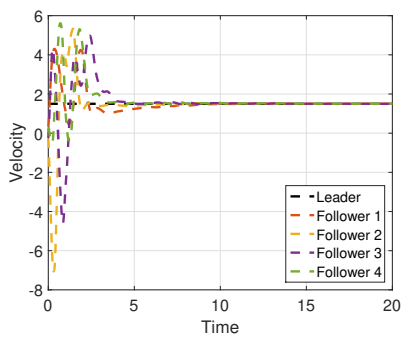

(b)
Fig. 5: Leader following consensus with a moving leader (a) position consensus - (b) velocity consensus

between two agents is given in Figure 7. 

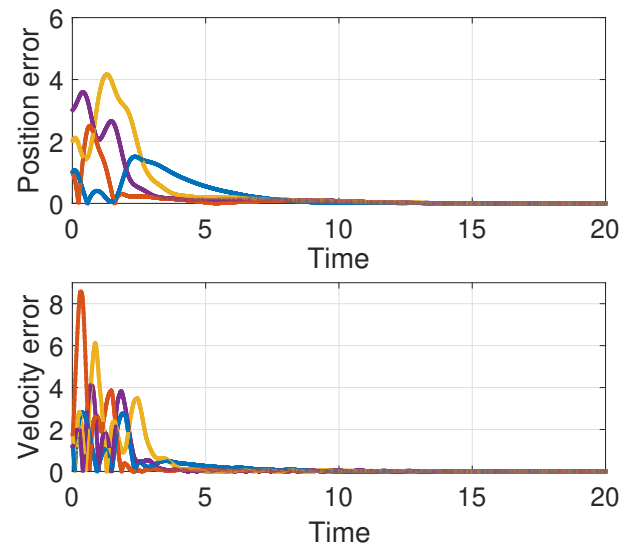

Fig. 6: Tracking errors for consensus with a moving leader

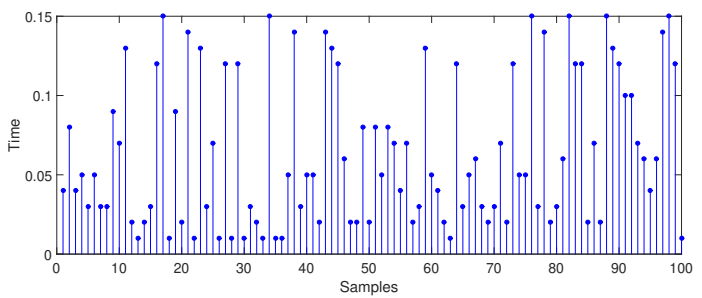

Fig. 7: Sampling periods for data transmission between the leader and follower 1

\section{CONCLUSION}

In this paper, we study the problem of leader-following consensus of second-order MAS with switching topology. The agents cannot measure their velocity and acceleration. They only share their position state with neighbors in a discrete aperiodic and asynchronous way. Using an ADT approach, sufficient conditions are derived through Lyapunov-based stability analysis to design an observer-based control protocol which solves the leader-following consensus. The investigation of the case where individual graphs do not necessarily have a spanning tree but only a joint-graph containing a spanning tree in a time period is considered for future work.

\section{REFERENCES}

[1] S. A. Ajwad, E. Moulay, M. Defoort, T. Ménard, and P. Coirault, "Output-feedback formation tracking of second-order multi-agent systems with asynchronous variable sampled data," in 2019 IEEE 58th Conference on Decision and Control (CDC). IEEE, 2019, pp. 44834488.

[2] P.-A. Bliman and G. Ferrari-Trecate, "Average consensus problems in networks of agents with delayed communications," Automatica, vol. 44, no. 8, pp. 1985-1995, 2008.

[3] Z. Qu, J. Wang, and R. A. Hull, "Cooperative control of dynamical systems with application to autonomous vehicles," IEEE Transactions on Automatic Control, vol. 53, no. 4, pp. 894-911, 2008.

[4] J. Zhu, Y.-P. Tian, and J. Kuang, "On the general consensus protocol of multi-agent systems with double-integrator dynamics," Linear Algebra and its Applications, vol. 431, no. 5-7, pp. 701-715, 2009.

[5] W. Yu, G. Chen, and M. Cao, "Some necessary and sufficient conditions for second-order consensus in multi-agent dynamical systems," Automatica, vol. 46, no. 6, pp. 1089-1095, 2010.

[6] W. Zhu and D. Cheng, "Leader-following consensus of second-order agents with multiple time-varying delays," Automatica, vol. 46, no. 12, pp. 1994-1999, 2010
[7] B. Tian, H. Lu, Z. Zuo, and W. Yang, "Fixed-time leader-follower output feedback consensus for second-order multiagent systems," IEEE transactions on cybernetics, vol. 49, no. 4, pp. 1545-1550, 2018.

[8] H. Pan and W. Qiao, "Consensus of double-integrator discrete-time multi-agent system based on second-order neighbors' information," in The 26th Chinese Control and Decision Conference (2014 CCDC). IEEE, 2014, pp. 1946-1951.

[9] Y. Cao and W. Ren, "Multi-vehicle coordination for double-integrator dynamics under fixed undirected/directed interaction in a sampled-data setting," International Journal of Robust and Nonlinear Control: IFACAffiliated Journal, vol. 20, no. 9, pp. 987-1000, 2010.

[10] X. Xu, S. Chen, and L. Gao, "Observer-based consensus tracking for second-order leader-following nonlinear multi-agent systems with adaptive coupling parameter design," Neurocomputing, vol. 156, pp. 297-305, 2015.

[11] W. Yu, W. X. Zheng, G. Chen, W. Ren, and J. Cao, "Second-order consensus in multi-agent dynamical systems with sampled position data," Automatica, vol. 47, no. 7, pp. 1496-1503, 2011.

[12] N. Huang, Z. Duan, and G. R. Chen, "Some necessary and sufficient conditions for consensus of second-order multi-agent systems with sampled position data," Automatica, vol. 63, pp. 148-155, 2016.

[13] J. Zhan and X. Li, "Consensus in networked multiagent systems with stochastic sampling," IEEE Transactions on Circuits and Systems II: Express Briefs, vol. 64, no. 8, pp. 982-986, 2016.

[14] S.-L. Du, W. Xia, W. Ren, X.-M. Sun, and W. Wang, "Observerbased consensus for multiagent systems under stochastic sampling mechanism," IEEE Transactions on Systems, Man, and Cybernetics: Systems, vol. 48, no. 12, pp. 2328-2338, 2017.

[15] M. Farza, M. M'Saad, M. L. Fall, E. Pigeon, O. Gehan, and K. Busawon, "Continuous-discrete time observers for a class of mimo nonlinear systems," IEEE Transactions on Automatic Control, vol. 59, no. 4, pp. 1060-1065, 2014.

[16] T. Menard, E. Moulay, P. Coirault, and M. Defoort, "Observer-based consensus for second-order multi-agent systems with arbitrary asynchronous and aperiodic sampling periods," Automatica, vol. 99, pp. 237 $245,2019$.

[17] S. A. Ajwad, T. Menard, E. Moulay, M. Defoort, and P. Coirault, "Observer based leader-following consensus of second-order multi-agent systems with nonuniform sampled position data," Journal of the Franklin Institute, vol. 356, no. 16, pp. 10031-10057, 2019.

[18] G. Casadei, A. Isidori, and L. Marconi, "About disconnected topologies and synchronization of homogeneous nonlinear agents over switching networks," International Journal of Robust and Nonlinear Control, vol. 28, no. 3, pp. 901-917, 2018.

[19] Y. Cai, H. Zhang, K. Zhang, and Y. Liang, "Distributed leader-following consensus of heterogeneous second-order time-varying nonlinear multiagent systems under directed switching topology," Neurocomputing, vol. 325, pp. 31-47, 2019.

[20] Q. Song, F. Liu, J. Cao, and W. Yu, "Pinning-controllability analysis of complex networks: an m-matrix approach," IEEE Trans. on Circuits and Systems, vol. 59, no. 11, pp. 2692-2701, 2012.

[21] H. Zhang, Z. Li, Z. Qu, and F. L. Lewis, "On constructing lyapunov functions for multi-agent systems," Automatica, vol. 58, pp. 39-42, 2015.

[22] J. P. Hespanha and A. S. Morse, "Stability of switched systems with average dwell-time," in Proceedings of the 38th IEEE conference on decision and control (Cat. No. 99CH36304), vol. 3. IEEE, 1999, pp. 2655-2660.

[23] A. Bédoui, M. Farza, M. M'Saad, and M. Ksouri, "Robust nonlinear controllers for bioprocesses," IFAC Proceedings Volumes, vol. 41, no. 2 , pp. 15 541-15 546, 2008.

[24] J.-P. Gauthier, H. Hammouri, S. Othman et al., "A simple observer for nonlinear systems applications to bioreactors," IEEE Transactions on automatic control, vol. 37, no. 6, pp. 875-880, 1992.

[25] Y. Cao, W. Yu, W. Ren, and G. Chen, "An overview of recent progress in the study of distributed multi-agent coordination," IEEE Transactions on Industrial informatics, vol. 9, no. 1, pp. 427-438, 2012.

[26] X. Ge, Q.-L. Han, D. Ding, X.-M. Zhang, and B. Ning, "A survey on recent advances in distributed sampled-data cooperative control of multi-agent systems," Neurocomputing, vol. 275, pp. 1684-1701, 2018.

[27] K. Chen, J. Wang, Y. Zhang, and Z. Liu, "Second-order consensus of nonlinear multi-agent systems with restricted switching topology and time delay," Nonlinear Dynamics, vol. 78, no. 2, pp. 881-887, 2014.

[28] L. Gao, B. Xu, J. Li, and H. Zhang, "Distributed reduced-order observerbased approach to consensus problems for linear multi-agent systems," IET Control Theory \& Applications, vol. 9, no. 5, pp. 784-792, 2015. 